\title{
Event Charts for the Analysis of Adverse Events in Longitudinal Studies: An Example from a Smoking Cessation Pharmacotherapy Trial
}

\author{
Joel A. Dubin ${ }^{*}, 1$ and Stephanie S. O’Malley ${ }^{2}$ \\ ${ }^{I}$ Department of Statistics \& Actuarial Science, Department of Health Studies \& Gerontology, University of Waterloo, \\ Waterloo, ON, Canada \\ ${ }^{2}$ Department of Psychiatry, Yale University, New Haven, CT, USA
}

\begin{abstract}
Purpose: To illustrate the use of one particular graphical method, the event chart (Lee et al., 2000), for the display of adverse events (AE's), along with other important considerations such as time on treatment/intervention, severity of AE's, treatment assignment, gender, etc., in longitudinal studies. These graphs can also include other key information such as efficacy measures and time-dependent covariates of interest.

Methods: Emphasizing an application of a dose-ranging smoking cessation trial of naltrexone, we use event charts to convey a few potentially interesting findings from the complex data from this trial, with particular attention paid to the analysis of the safety (AE) data from the subset of individuals who dropped out before the end of the treatment phase of the study.

Results: The event charts conveyed some interesting findings regarding relationships between gender, AE's and dropout time, as well as between treatment group, AE's and dropout time, and between AE burden and dropout time.

Conclusion: Event charts can be one of the helpful exploratory tools in investigating the pattern of adverse events and their possible association with covariates and time on treatment/intervention in longitudinal studies. Findings from the event chart analysis of AE's could potentially lead to more formal statistical analysis and modeling. Software for generating these event charts is available in $\mathrm{R}$ and S-Plus.
\end{abstract}

Keywords: Dropout, event chart, exploratory analysis, graphical analysis, naltrexone, nicotine patch, side effects.

\section{INTRODUCTION}

Analysis tools for complex data need to balance the ability to uncover meaningful results while simultaneously communicating those results in as straightforward manner as possible. Graphical tools can be one approach toward achieving this balance. One such tool is the event chart [1], where multiple types of "events" over time can be plotted on a unique single line for each person participating in the study, each line stacked horizontally, with time plotted on the $\mathrm{x}$-axis. The lines can then be sorted to potentially uncover interesting associations and relationships from longitudinal studies. The events plotted will be studyspecific. For example, in a bladder cancer treatment study, where recurrences of bladder cancer are common, each person would have a bladder cancer occurrence indicated by the same mark, some people would have multiple appearances of the mark over time, and distinct marks could be used each for death and other forms of participant dropout, as well as Type I (end-of-study) censoring.

In the bladder cancer example, one interesting event chart would be to first sort the participants by treatment group, then by descending number of cancer occurrences. It could

\footnotetext{
*Address correspondence to this author at the Department of Statistics and Actuarial Science, University of Waterloo, 200 University Ave W., Waterloo, ON, N2L 3G1, Canada; Tel: 1-519-885-1211, Ext. 37318; Fax: 1519-746-1875; E-mail: jdubin@uwaterloo.ca
}

be that each group has roughly the same percentage of individuals with at least one cancer but the control group might have a greater number of recurrent events. An example event chart, taken from a bladder cancer trial analyzed in [2], can be found in Fig. (1), where the graphical evidence suggests a greater number of patients without recurrence in the thiotepa arm (Arm 2), a greater number with four recurrences in the placebo arm (Arm 1), and a faster time to dropout for those with a larger number of recurrences in the thiotepa arm, possibly due to a combination of poor results with the experience of side effects of the active treatment.

Assuming the data are available, certainly more complex relationships can be investigated (e.g., roles of treatment compliance, prior cancer history, family history, genetic data, etc.) with event charts. Though modeling can be a powerful tool for complex data such as that coming from recurrent events, graphs can often tell an important (and informative) story, including to those unfamiliar with complex survival and longitudinal models. In addition, the graphs can potentially serve as an exploratory tool for subsequent statistical modeling.

Many datasets possess an equal level of complexity or greater of recurrent event outcomes, for which graphical methods such as the event chart could prove useful. Another such dataset comes from an investigation of adverse events in a smoking cessation trial. Specifically, O'Malley et al. [3] 


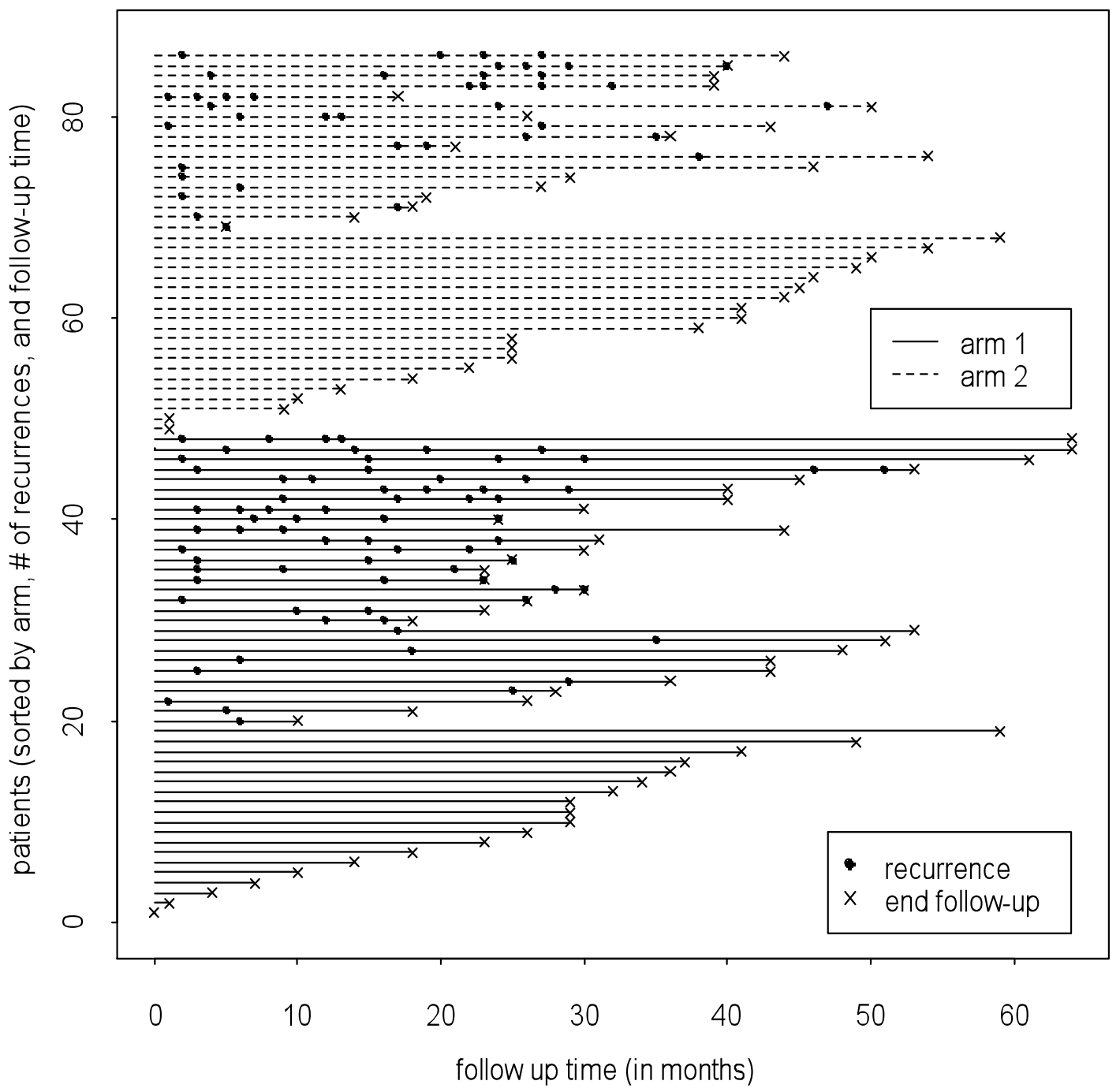

Fig. (1). Patients $(n=86)$ sorted by three variables (treatment arm: $1=$ placebo, 2 = thiotepa; number of bladder cancer recurrences; patient follow-up time).

investigated the use of naltrexone, a nonaddicting opioid antagonist, combined with nicotine patch on participants who were interested in quitting smoking cigarettes. This was a dose-ranging study, with all participants receiving patch, but with participants randomized to a $0,25,50$, or $100 \mathrm{mg}$ daily dose of naltrexone. Aside from looking at the efficacy of naltrexone on smoking cessation and finding an optimal dose, there was interest in investigating the relationship between adverse events experienced by the participants and dropout, and if this relationship was influenced by treatment regimen and other factors. In this paper, event charts will be presented for this investigation.

Graphical methods are recommended for the analysis of adverse events (AE's) from clinical trials. For example, in a still-relevant commentary, Levine and Szarfman [4] discussed the important need for using graphical approaches to the analysis of safety data. They also presented some basic graphical tools for investigating AE's in clinical trials, including a delta plot that is one type of event chart, where the $y$-axis is not time (as in the example in Fig. (1)) but instead a physiological value of interest; the initial and future values for each patient are displayed in the delta plot and the graph is sorted by descending change. In a proceedings article, Hsu et al. [5] presented unsorted event charts for safety (i.e., AE) data, where the $\mathrm{x}$-axis represented time. We suggest that additional manipulation is necessary on these earlier versions of event charts for $\mathrm{AE}$ data in order to provide additional features and associations that may be useful for descriptive purposes as well as for subsequent modeling of the data.

A more recent article [6] provides a variety of displays for the analysis of safety data from clinical trials, including informative use of boxplots, distribution plots (e.g., distribution of the maximum level of a toxicity screening test), cumulative distribution plots, and multidimensional plots.

In this article, we would like to build upon what has been done previously graphically for the display of AE's in longitudinal studies, including clinical trials, and apply these methods to the aforementioned pharmacotherapy smoking cessation trial. As identification of AE's has become such a focus in medical research, we hope that event charts will be used to help illuminate such possible concerns that arise in longitudinal studies. 
This paper is organized as follows. In Methods, we further describe the naltrexone study, discuss how AE's were recorded in the study database, and state the goals of the graphical analysis of AE's. In Results, we show a variety of event charts for displaying the AE data from the naltrexone study. In Discussion, we summarize the results, talk about further possible investigation of the naltrexone study data, and highlight the greater role that event charts can play in the study of AE's in longitudinal studies.

\section{METHODS}

\section{The Naltrexone Study}

O'Malley et al. [3] conducted a dose-ranging study of naltrexone as an augmentation strategy of nicotine patch for the purposes of aiding in smoking cessation of individuals interested in quitting. Four hundred participants were enrolled and randomized to one of four treatment groups. In each group, the participants received $21 \mathrm{mg}$ transdermal nicotine patch (Nicotine CQ, GlaxoSmithKline) for six weeks, beginning on their quit date, as well as weekly behavioral counseling. Then, depending on treatment assignment, participants received one of four doses of naltrexone in pill form, i.e., $0 \mathrm{mg}, 25 \mathrm{mg}, 50 \mathrm{mg}, 100 \mathrm{mg}$, daily. Dose reductions of one half were permitted once based on lack of tolerability. Those who continued not to tolerate naltrexone were permitted to remain in the behavioral treatment and to continue on patch. This research was conducted at the Connecticut Mental Health Center

Of the 400 randomized, 385 actually received treatment, and this is the cohort used in the analysis. On a weekly basis, both at the quit date (week 0) and for the subsequent six weeks while on treatment (weeks 1 through 6), participants were assessed on smoking status, adverse events, withdrawal symptoms, craving status, and other measurements of interest such as weight.

\section{Recording of Adverse Events}

The primary consideration for the graphical analysis in this paper is the adverse events (AE's) experienced by the participants. Information on AE's was obtained using a checklist that included the commonly reported events for naltrexone (e.g., nausea) and for transdermal nicotine patch (e.g., rash), with severity rated as 0 (not present), 1 (mild), 2 (moderate), or 3 (severe). In addition, participants were asked to report any additional concerns. This information was collected prior to beginning treatment (week 0) and at each of the six weekly follow-up appointments during the treatment phase of the study (weeks 1 through 6). It will be the AE's on the checklist that will be our basis of analysis here. More specifically, we will focus upon the moderate and severe AE's, since, likely due to the list prompting of specific types of AE's, almost all individuals reported mild AE's during the course of treatment. Liver function tests were also obtained at certain appointments to monitor hepatotoxicity, but these tests will not be studied in this paper.

\section{Analysis}

The entire analysis presented here will be exploratory using a graphical approach, with the focus on different event charts showing experiences of moderate and severe AE's. As the primary goal of this paper is to communicate the ability of event charts to elucidate potentially interesting and relevant details of an $\mathrm{AE}$ data analysis from longitudinal studies, including clinical trials, we will, without loss of generality, limit the current analysis to a subset of participants in the study. Specifically, we will display the graphs for those who dropped out of the study prior to the end of the six-week treatment period and investigate the relationship between $\mathrm{AE}$ experience and dropout, where early dropout in this article means the participant made it through just 37 days or fewer of the 42-day (6-week) treatment period. The analysis strategy is to display a variety of event charts presenting the AE results, in general ascending order of detail, with accompanying commentary on the different results that can be observed in each graph.

\section{RESULTS}

For illustrative purposes, we will combine the moderate and severe reporting of adverse events (AE's) in any given week for a given participant. One, possibly overly simplistic, approach is to dichotomize individuals in a given week into those who had at least one moderate or severe AE vs those who did not. As mentioned in Methods, we will focus all of the following graphs on those who dropped out of the study prior to the end of the six week treatment period, where, again, for the purposes of this article, early dropout signifies the participant made it through only 37 days or fewer of the 42-day (6-week) treatment period. This subsample included 78 (or 20.3\%) of the 385 participants who received treatment.

The first of the event charts for the smoking cessation study, Fig. (2), presents the most simplistic of AE event chart graphs. Though it is not very informative, it is a good starting point from which to build more interesting graphs. Specifically, Fig. (2) shows the 78 participants who dropped out and provides marks (black dots) in any week in which at least one moderate or severe $\mathrm{AE}$ was reported, as well as a mark (an "X") for the participant's drop out date. The type of AE's are not distinguished here (say, nausea $v$ seadache $v s$ depression, etc., or possibly by hypothesized reason for $\mathrm{AE}$, say due to naltrexone $v s$ patch $v s$ withdrawal symptom $v s$ combination of reasons) but could easily be done so with appropriate information in the dataset. The graph is unsorted on the y-axis, at least in terms of a covariate of interest; it only represents the dropout individuals in the order at which they were enrolled in the study.

There are a variety of individuals in Fig. (2), including those who dropped out early with no AE marks, those who experienced just one week of at least one AE, and those with multiple weeks of AE's. A line for a given individual simply means he/she experienced a moderate or severe $\mathrm{AE}$ on at least one week prior to their dropout date. There are also some individuals who experienced at least one moderate or severe $\mathrm{AE}$ prior to even receiving treatment (on Day 0 , which was the quit date). These AE's on Day 0 are obviously not due to treatment or patch use, but may be due to withdrawal symptoms such as headache (for those who quit prior to Day 0), or anxiety due to consideration of upcoming days without smoking and/or with treatment usage, etc.

Fig. (3) improves the readability of Fig. (2), by simply sorting the vertical axis by time until dropout. This allows a 


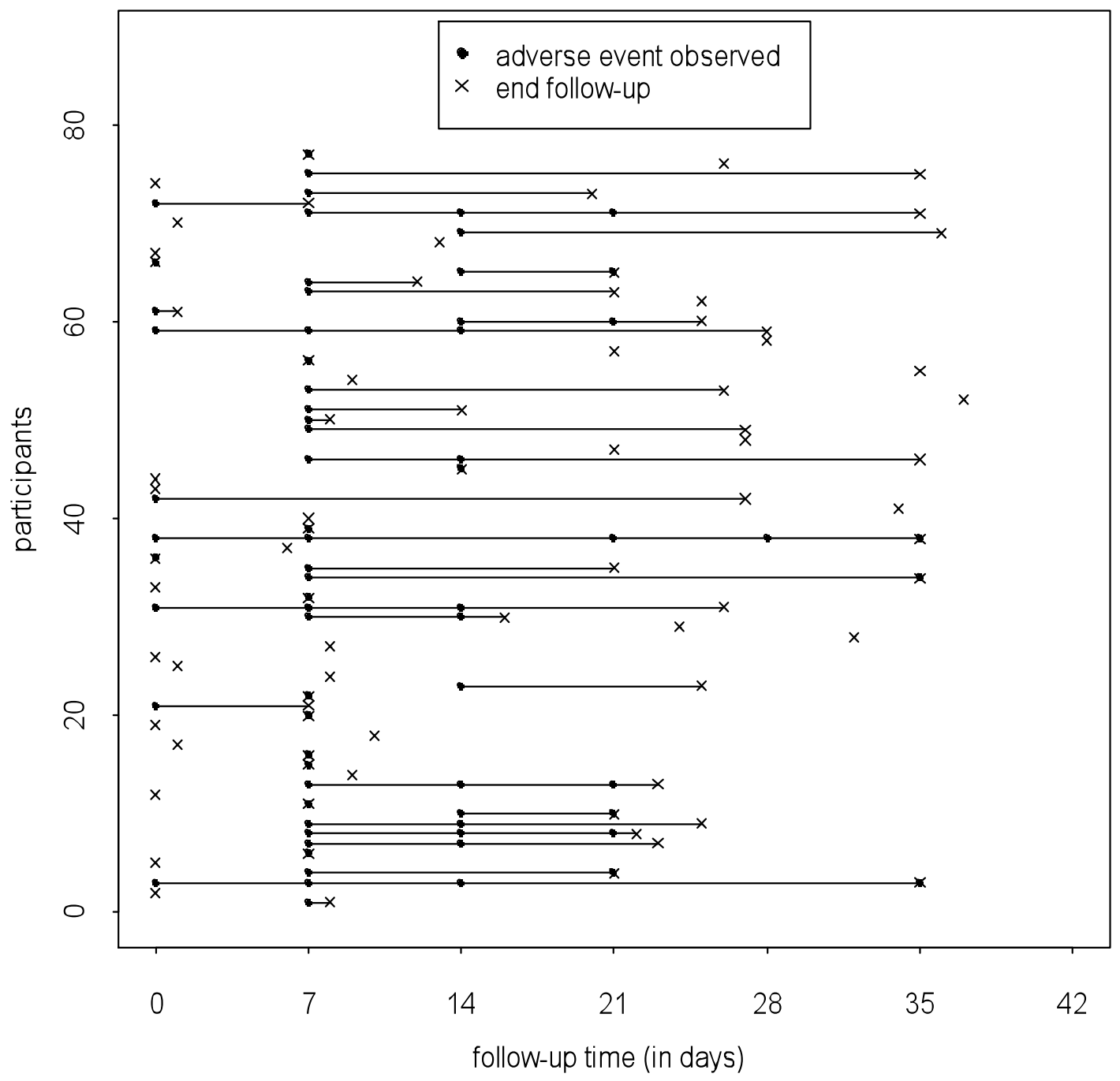

Fig. (2). Event chart showing adverse event (AE) occurrence and final date of observation for $\mathrm{n}=78$ dropouts in naltrexone plus nicotine patch smoking cessation study. The vertical axis is unsorted by any covariate, reflecting only order of entry into study. All participants begin at Day 0. A horizontal line for a given individual simply means he/she experienced a moderate or severe AE on at least one week prior to their dropout date.

much easier way to view the distribution of dropout cases. In particular, we can observe that about $1 / 2$ of the dropouts never made it back to their week 2 visit. This type of graph also allows us to look at associations between when someone dropped out and AE experience. For example, the large majority of those who dropped out prior to Week 2 (Day 14) experienced at least one moderate or severe AE at Week 1, whereas the percentage of those with AE's in the week prior to a later dropout is not as high, possibly suggesting dropout for these individuals was due to reasons other than recent AE's.

A potentially more revealing event chart than that in Fig. (3) can be seen in Fig. (4). This is a replica of Fig. (3), except now the marks displayed in the figure represent "AE burden", where this burden is represented proportionally by the number (i.e., sum) of distinct moderate or severe AE's a given participant reported in a given week. It appears evident, that among the participants who dropped out, those experiencing heavy burden (i.e., with the larger symbols) dropped out in a shorter period of time after that experience versus those experiencing a lower number of moderate or severe AE's. This would seem to make intuitive sense for this study population, but the display provides apparent evidence of this association. Note that for a more complete understanding of the association of AE burden with dropout, we would need to also look at the AE experience of those who remained in the study. The analysis of treatment completers is not considered here, but is a focus of future work.

Fig. (5) is the same as Fig. (3) except now we have sorted on gender prior to sorting by time to dropout. There are a couple of interesting aspects of this graph. First, there is an apparent trend that men are more likely to dropout of the study than women. However, among the women, a larger percentage drops out by the week 2 (day 14) visit than among the men. This is the type of observation that should be investigated further, to see, for example, if a problem exists with the initial tolerance (of naltrexone, patch, and/or withdrawal) for women or if there are other reasons (e.g., weight gain) for this trend. Nonetheless, as mentioned above, 


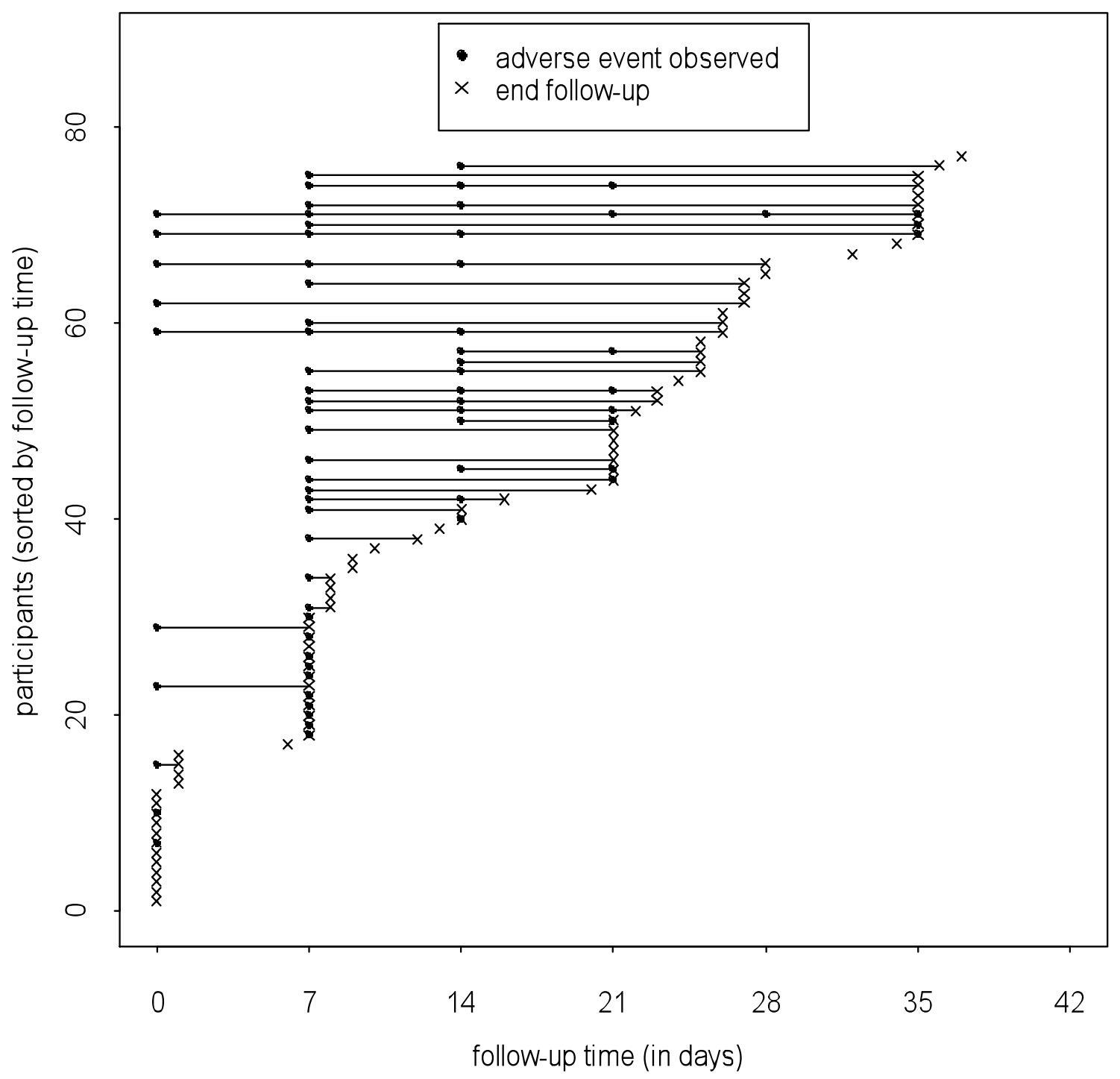

Fig. (3). Event chart showing adverse event occurrence and final date of observation for $n=78$ dropouts in naltrexone plus nicotine patch smoking cessation study. The vertical axis is sorted by ascending time until dropout. All participants began at Day 0 . A horizontal line for a given individual simply means he/she experienced a moderate or severe AE on at least one week prior to their dropout date.

it is men who drop out more often, and further investigation for this would also likely be warranted.

The final figure presented here, Fig. (6) is similar to Fig. (3), except we have now sorted the graph by treatment prior to sorting by time to dropout. A trend is apparent where there are a higher number of dropouts as the dosage of naltrexone is increased. In addition, it is interesting that among those in the highest naltrexone arm $(100 \mathrm{mg})$, it is evident that a number left the study very early, though there are those who persevered through some early AE experience to stay in the study for several weeks. This may be due to a combination of a few of these participants having their doses lowered due to tolerance issues, resolution of adverse events, as well as possibly detecting some success on the drug. This differs from the $0 \mathrm{mg}$ arm, where fewer dropouts occurred, but among these dropouts, the participants did not stay in the study very long. A lack of efficacy could very well be the reason for the $0 \mathrm{mg}$ arm dropouts. Obviously, further investigation is required; these various event charts, particularly in Figs. (3-6), provide a good exploratory basis for which to further investigate such questions.

Overall, the graphical analysis we have taken here provides fundamentally distinct information from the more standard tabular analysis undertaken in the original paper reporting the results of the naltrexone trial [3]. In that tabular analysis, the focus was on differential participant reporting of specific AE's across treatment groups (e.g., headache, nausea, dizziness, itching, etc.), without consideration of in which weeks the AE's were reported. The event chart analysis instead allows for a time-specific reporting of events, and could serve as a complement to the tabular approach. As seen in the figures in this paper, event charts will also allow for consideration of time on study and multiple covariates, whereas a tabular analysis considering all these factors would generally become too cumbersome. 


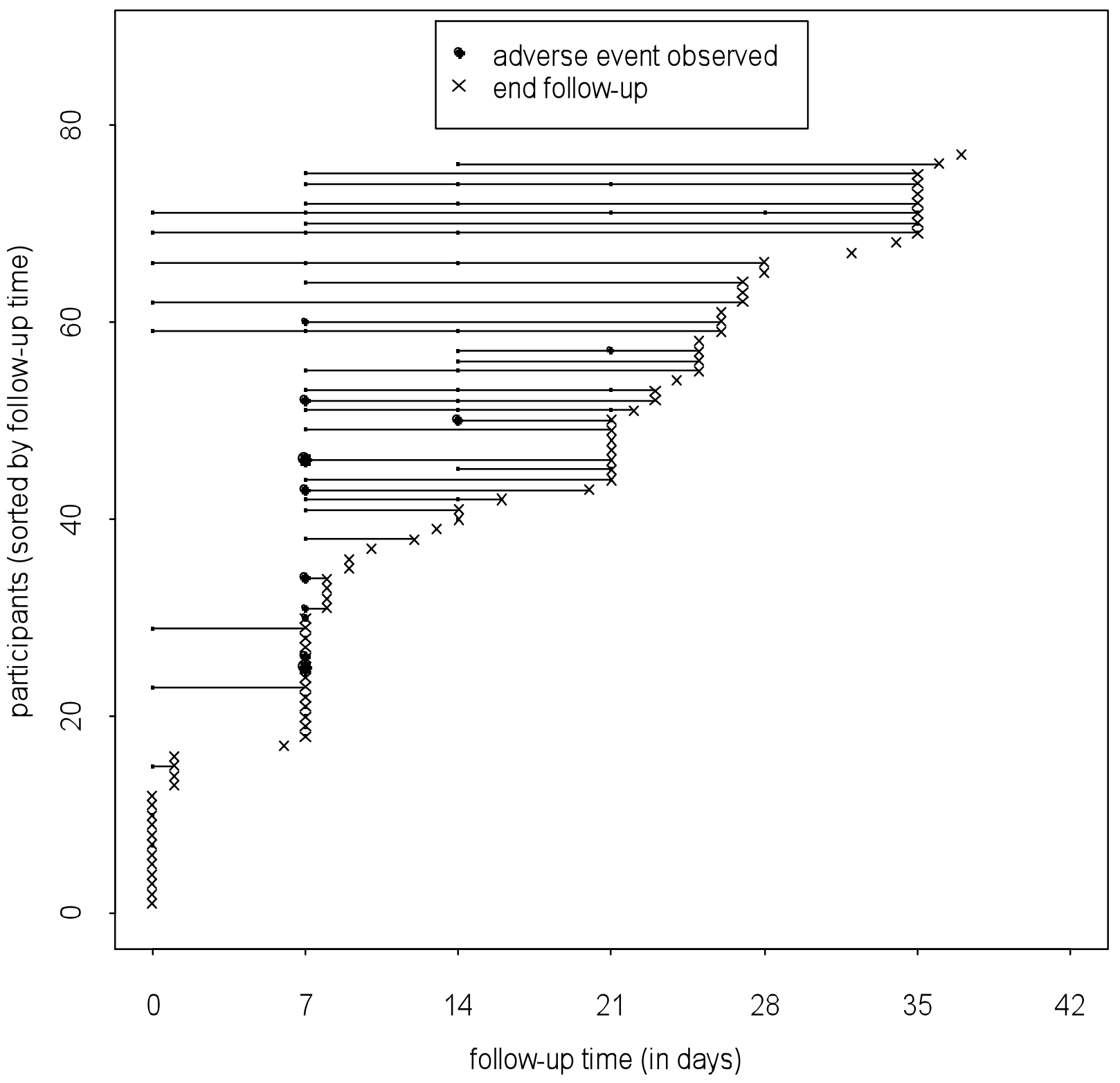

Fig. (4). Event chart showing adverse event occurrence and burden (shown by size of symbol) and final date of observation for $\mathrm{n}=78$ dropouts in naltrexone plus nicotine patch smoking cessation study. The vertical axis is sorted by ascending time until dropout. All participants began at Day 0. The size of the dot on any day reflects "AE burden", here measured as the number (sum) of distinctly recorded moderate or severe AE's reported by the participant in the past week (e.g., burden $=2$, if one severe case of nausea and one moderate case of headache was reported in the past week). A horizontal line for a given individual simply means he/she experienced a moderate or severe AE on at least one week prior to their dropout date.

\section{DISCUSSION}

Our goal for this paper was to demonstrate the potential usefulness of event charts [1] for displaying adverse events (AE's) experienced by participants in longitudinal studies, including clinical trials, as well as looking for potential associations between AE's and covariates and/or experiences such as dropout patterns. We utilized only a subset of participants (i.e., those who dropped out before the end of the treatment period) from a pharmacotherapy smoking cessation trial for illustrative purposes, but we firmly believe that event charts will be helpful in discovering important aspects of AE's for many different types of longitudinal studies and participants therein. In the pharmacotherapy study itself, we detected some interesting patterns of AE's for those who dropped out of the study early, including the timing and burden of AE's in relationship to dropout, as well as differing patterns of dropout by gender and treatment group and possibly differing patterns of $\mathrm{AE}$ experience within these different strata.

The flexibility of allowing different marks for different AE's and/or other events of interest (e.g., dropout, compliance indicators, occurrence of various competing risks, etc.) as well the sorting of the plots by various covariates of interest makes the event chart a potentially important component in the analysis of AE's in longitudinal studies. Further analysis of the smoking cessation study could include investigating the relationship between AE's, compliance, treatment and dropout, both with the event 


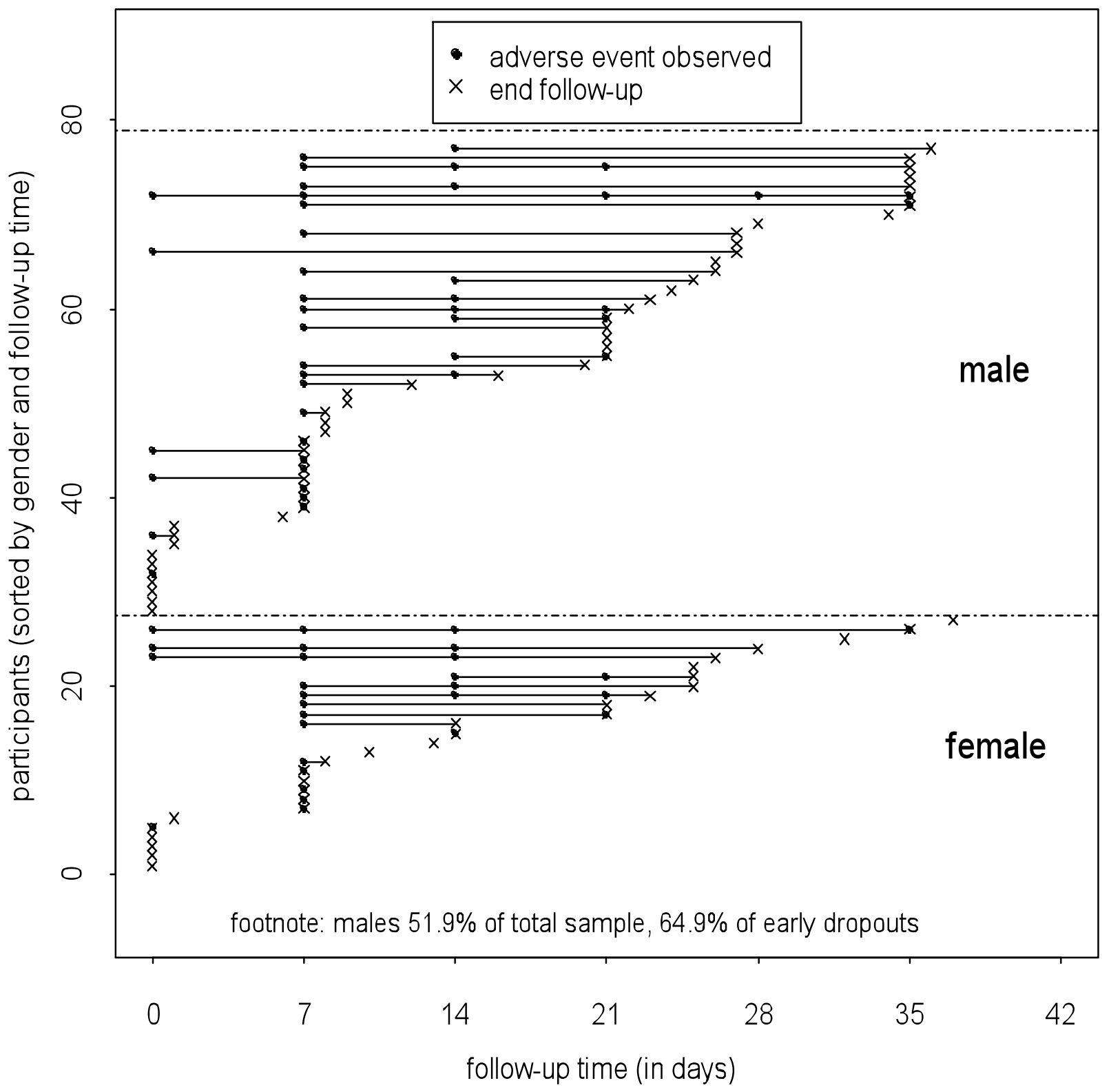

Fig. (5). Event chart showing adverse event occurrence and final date of observation for $\mathrm{n}=78$ dropouts in naltrexone plus nicotine patch smoking cessation study. The vertical axis is sorted by both gender and ascending time until dropout. All participants began at Day 0. A horizontal line for a given individual simply means he/she experienced a moderate or severe AE on at least one week prior to their dropout date.

charts as well as through modeling. Certainly, it is important to confirm any results as suggested in any exploratory analysis, such as done here with the event charts. In addition, if we wanted to consider a larger number of participants using event charts, for example, to plot all 385 original participants who received treatment in the naltrexone trial, one could see if the markings are too tight for a single chart, or instead consider multiple charts via subsetting by covariates of interest such as treatment group.

Some recent work has been published describing complex hierarchical and multivariate time-to-event modeling of adverse events from clinical trials (e.g., [7, 8]) or modeling focusing on the relationship between $\mathrm{AE}$ experience and informative dropout [9]. This is important work and hopefully will allow for a better understanding of the role that AE's play in longitudinal studies, including clinical trials. We also want to advocate for the use of event charts as a descriptive graphical approach to complement formal statistical analysis of adverse events in such studies, as event charts can serve as useful tools for illustrating the nature of various aspects of a given dataset and for hypothesis generation. As Levine and Szarfman [4] point out, "We have found that visual displays are invaluable in understanding safety data". Event charts, as described and illustrated here, can serve to fulfill this role.

NOTE: There is a function called event.chart, which has been made available in Frank E. Harrell's Hmisc package in R (R Core Development Team, 2010) and Hmisc library in S-Plus ${ }^{\circledR}$. All event charts presented in this paper were created using event.chart (in R), and the code using these functions 


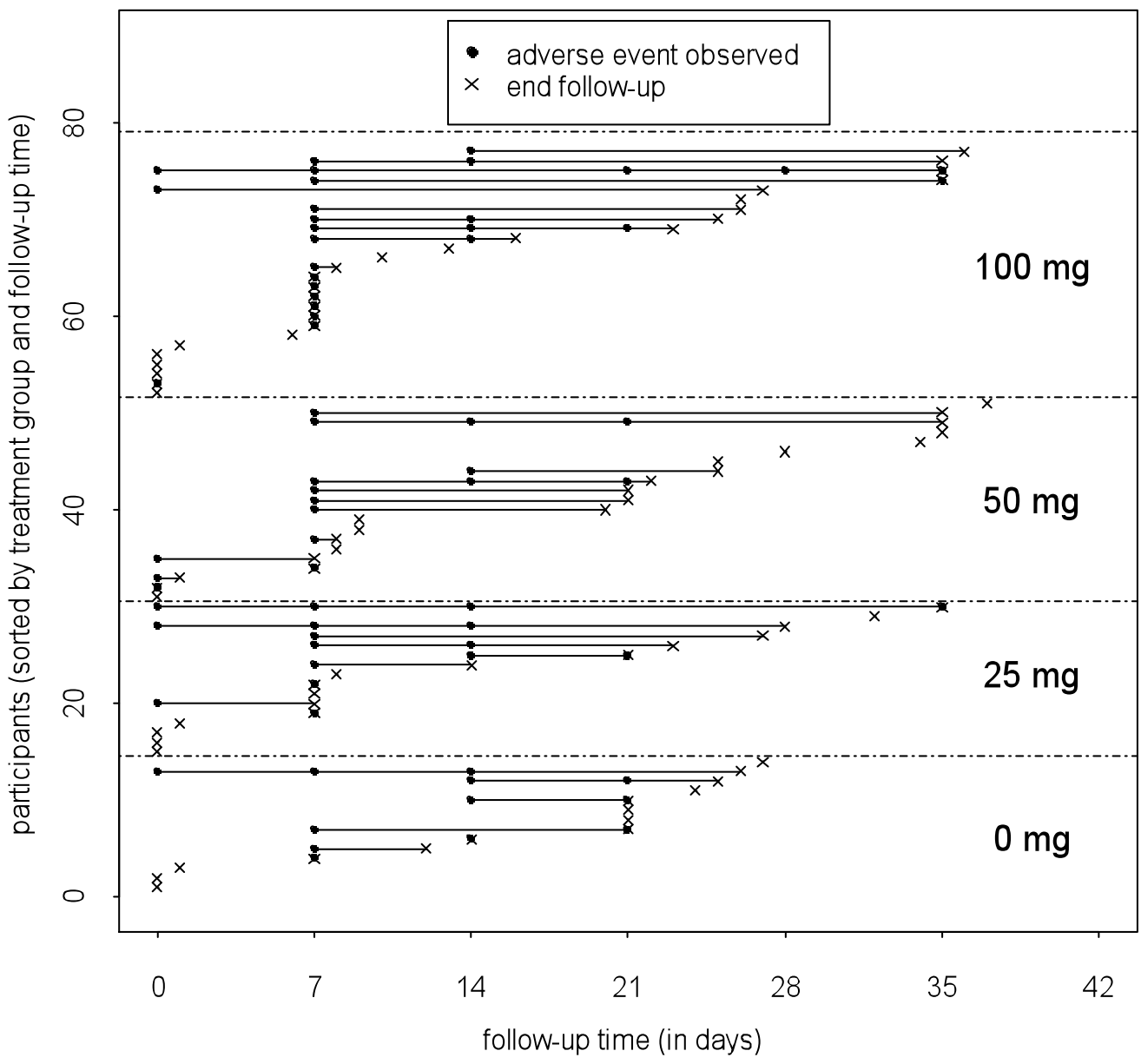

Fig. (6). Event chart showing adverse event occurrence and final date of observation for $\mathrm{n}=78$ dropouts in naltrexone plus nicotine patch smoking cessation study. The vertical axis is sorted by both treatment group (patch plus either 0, 25, 50, or 100 mg of naltrexone) and ascending time until dropout. All participants began at Day 0. A horizontal line for a given individual simply means he/she experienced a moderate or severe $\mathrm{AE}$ on at least one week prior to their dropout date.

to create these graphs can be obtained from the primary author of this article.

\section{ACKNOWLEDGEMENTS}

This research was supported in part by NIH grants K05AA014715, P50-DA13334, P50-AA15632 and by the State of Connecticut, Department of Mental Health and Addictions Services, as well as NSERC Discovery Grant RGPIN 32709306. The content is solely the responsibility of the authors and does not necessarily represent the official views of the National Institute on Alcohol Abuse and Alcoholism, the National Institute on Drug Abuse, or the National Institutes of Health.

\section{REFERENCES}

[1] Lee JJ, Hess KH, Dubin JA. Extensions and applications of event charts. Am Stat 2000; 54: 63-70.

[2] Wei LJ, Lin DY, Weissfeld L. Regression-analysis of multivariate incomplete failure time data by modeling marginal distributions. J Am Stat Assoc 1989; 84: 1065-73.
[3] O'Malley SS, Cooney J, Krishnan-Sarin S, et al. A controlled trial of naltrexone augmentation of nicotine replacement therapy for smoking cessation. Arch Int Med 2006; 166: 667-74.

[4] Levine JG, Szarfman A. Standardized Data Structures and Visualization Tools: A way to accelerate the regulatory review of the integrated summary of safety of new drug applications. Biopharm Rep 1996; 4: $12-7$.

[5] Hsu C, Zhou Z, Hardin JM. A useful chart to display adverse event occurrences in clinical trials. Proceedings of the $27^{\text {th }}$ SAS Users Group International Conference 2002; paper 119-27.

[6] Amit O, Heiberger RM, Lane PW. Graphical approaches to the analysis of safety data from clinical trials. Pharm Stat 2008; 7: 20-35.

[7] Berry SM, Berry DA. Accounting for multiplicities in assessing drug safety: a three-level hierarchical mixture model. Biometrics 2004; 60 : 418-26.

[8] Guttner A, Kubler J, Pigeot I. Multivariate time-to-event analysis of multiple adverse events of drugs in integrated analyses. Stat Med 2007; 26: $1518-31$.

[9] Nishikawa M, Tango T, Ogawa M. Non-parametric inference of adverse events under informative censoring. Stat Med 2006; 25: 39814003. 